\title{
Does short-term endotracheal intubation affects the acoustic characteristics?
}

Toran KC ${ }^{1}$, BK Lal ${ }^{2}$

${ }^{1}$ Associate Professor, ${ }^{2}$ Speech Language Pathologist, Department of ENT-HNS, Kathmandu Medical College, Sinamangal, Kathmandu

\begin{abstract}
Introduction: Endotracheal intubation can produce various degrees of temporary and sometimes permanent damage to the laryngotracheal mechanism. Recent development of computer based voice analysis technology can now detect a minute changes in acoustic waveforms which a normal human ear cannot.

In the study we compared and analyzed the acoustic waveforms of 35 patients undergoing surgery under intubation anaesthesia.

Objective: The aim of the present series is to analyze the effects of short term intubation with computerized voice laboratory.

Materials and methods: Values of acoustic waveforms obtained from 35 patients were compared 48 hours after the short term endotracheal intubation anaesthesia. The comparisons were made in terms of perturbation (jitter and shimmer), harmonic- to noise ratio (HNR) and fundamental frequency $\left(\mathrm{F}_{0}\right)$.

Results: The pre-intubated voice characteristics when compared with the post-intubation group did not reveal any statistical difference $(\mathrm{P}>0.05)$. However, there was only a minimal decrease in $\mathrm{F}_{0}$.

Conclusion: The study revealed that, short term intubation anaesthesia does not alter the acoustic characteristics. The analysis of acoustic waveforms is a non invasive technique that helps to evaluate the effects of tracheal intubation on laryngeal function, a technique that warrants further evaluation.
\end{abstract}

Key words: Acoustic characteristics, Endotracheal intubation, Voice

G eneral anaesthesia nearly always requires endotracheal intubation for assisted ventilation and airway protection. Many patients, particularly the voice professionals always fear of their vocal changes following intubation anaesthesia, which is not uncommon either. This is primarily due to pressure injury or due to intubation trauma to the larynx rather than by central depression ${ }^{1,2,3}$. In another study it was observed that, changes in the acoustic waveforms of the larynx after intubation are caused by the mechanical impact of the tracheal tube, rather than by central depression 4 .

Though the consequences of short term laryngeal intubation are minor, and transient, the severity may range from mild dysphonia to tracheal stenosis ${ }^{2,3}$. Injuries secondary to the endotracheal intubation may occur at glottic level due to pressure exerted by the wall of the tube, and tracheal injury due to excessive pressure generated by the cuff or the tip, particularly in case of the prolong intubation ${ }^{5,6}$.
The perceptual evaluation of voice is a subjective assessment of the vocal characteristics and has significant limitations that can lead to confusion or disparity amongst the observers. Recent development of acoustic analysis technology can now access sustained sound waveform characteristic, which may not be detected by even a trained ear. In particular, changes in fundamental frequency $\left(\mathrm{F}_{0}\right)$, perturbation (jitter and shimmer) and harmonic-to noise ratio (HNR) have been shown to be significantly altered by various laryngeal pathology ${ }^{7}$. Researchers are on to delineate the extent to which voice waveform characteristics are altered by endotracheal intubation ${ }^{8,9}$. The study was undertaken to analyze the quality of voice following short term intubation anaesthesia and to compare the acoustic characteristics with the pre-operative data.

Correspondence

Dr. Toran $\mathrm{KC}$

Associate Professor

Department of ENT-HNS

Kathmandu Medical College

Sinamangal, Kathmandu

E-mail: torankc@yahoo.com 


\section{Material and methods}

A prospective study carried out from April 2008 on 35 patients selected from those who were scheduled for ear or sinonasal surgeries under general anaesthesia with intubation. There age ranged from 12 to 48 years with mean age of 26 years. Of the 35 cases 20 were males and 15 females. Patients with history of laryngeal surgeries or voice surgeries were not included in the study. These also included patients who had to undergo oropharyngeal surgeries which would have direct impact on the voice characteristics following the surgery.

All the patients underwent surgery under intubation anaesthesia and the duration of surgeries ranged from 30 minutes to 210 minutes with an average duration of 117 minutes. A variety of anaesthetic techniques were used, including different combination of anaesthetic medications and Polyvinylchloride(PVC) cuffed endotracheal tubes of suitable sizes ranging from number 6 to 8.5 were used.

All of them were analyzed perceptually before considering the acoustic analysis. Patients with GRBAS score ${ }^{10}$ more than ' 0 ' were not included in the study. The acoustic samples were recorded with Dr. Speech acoustic software (Tiger electronics, USA) on the day of admission one day prior to surgery. Second data were taken on the second post-operative day, i.e., after 48 hours of surgery. The four acoustic parameters were compared between the two groups, and their relation with the duration of surgery was also compared. Voice recording was done directly in their habitual loudness with a microphone placed at 30 centimetres from the patients' upper lip, and the person sitting in the comfortable position. Sustained vowel//i// was preferred instead of continuous speech ${ }^{11}$. The data were analyzed from mid 3 seconds of the vowel //i// and first $500 \mathrm{~ms}$ of the voice was discarded.

The data were analyzed in terms of perturbation (Jitter and shimmer), HNR and $\mathrm{F}_{0}$. The statistical analysis of the data was performed with SPSS 16.0 for windows. Differences were considered significant for $\mathrm{P}<0.05$.

\section{Results}

A total of 35 informed volunteer patients were involved in the study with 20 and 15 male and female patients respectively. Their pre-operative acoustic parameters viz., jitter, shimmer, HNR and fundamental frequency were compared and analysed following operation on the second post- operative day i.e., after 48 hours after surgery. All the patients had general anaesthesia with endotracheal intubation with duration ranging from 30 minutes to 210 minutes with average duration of 105 minutes. The four acoustic parameters thus analyzed between the two group of patients revealed no statistically significant difference of acoustic values $(\mathrm{P}>0.05)$, as depicted in the Table 1 . However, there was a minimum increment only in jitter. Shimmer and $\mathrm{F}_{0}$ were decreased, yet the paired t-test revealed that the inter group changes were not statistically different. Fundamental frequency $\left(\mathrm{F}_{0}\right)$ is the frequency of vocal fold vibration during the phonation. The pre-intubation mean $\mathrm{F}_{0}$ values were $231.77 \mathrm{~Hz}$ and $222.90 \mathrm{~Hz}$ in the post intubation group with a difference of $8.87 \mathrm{~Hz}(\mathrm{P}=0.135)$.

There were no changes in the HNR group either. These acoustic parameters were also analyzed with the duration of intubation anaesthesia and were subdivided between the two groups of either less than or more than 110 minutes. Data analysis with Fisher's exact test did not reveal any statistical difference between these two subgroups.

Table 1: Pre and Post Intubation Acoustic values, N: 35

\begin{tabular}{|l|c|c|c|c|c|c|c|c|}
\hline \multirow{2}{*}{} & \multicolumn{2}{|c|}{ Jitter(\%) } & \multicolumn{2}{c|}{ Shimmer(\%) } & \multicolumn{2}{c|}{ HNR(dB) } & \multicolumn{2}{c|}{ F $_{0}(\mathbf{H z})$} \\
\cline { 2 - 9 } & A & B & A & B & A & B & A & B \\
\hline Mean & 0.164 & 0.171 & 1.705 & 1.618 & 26.267 & 26.261 & 231.77 & 222.90 \\
\hline (SD) & $(0.05)$ & $(0.09)$ & $(0.85)$ & $(0.82)$ & $(4.72)$ & $(4.26)$ & $(68.16)$ & $(64.74)$ \\
\hline P-value* & \multicolumn{2}{|c|}{0.633} & \multicolumn{2}{|c|}{0.428} & \multicolumn{2}{|c|}{0.992} & \multicolumn{2}{c|}{0.135} \\
\hline
\end{tabular}

*Paired t-test applied. A: Pre operative, B: Post operative.

Table 2: Relation to duration with Surgery (N: 35)

\begin{tabular}{|c|c|c|c|c|c|}
\hline \multirow{2}{*}{ S. No. } & \multirow{2}{*}{\multicolumn{2}{|c|}{ Duration(in Mins.) }} & \multicolumn{2}{|c|}{ Median score (in mins.) } & \multirow{2}{*}{ p-Value* } \\
\hline & & & Less than 110 & More than 110 & \\
\hline \multirow[b]{2}{*}{1.} & Jitter(\%) & $<0.15$ & 9 & 5 & \multirow{2}{*}{0.491} \\
\hline & & $>0.15$ & 10 & 11 & \\
\hline \multirow{2}{*}{2.} & Shimmer(\%) & $<1.45$ & 10 & 8 & \multirow{2}{*}{1.0} \\
\hline & & $>1.45$ & 9 & 8 & \\
\hline \multirow{2}{*}{4.} & $\mathrm{HNR}(\mathrm{dB})$ & $<26.12$ & 9 & 8 & \multirow{2}{*}{1} \\
\hline & & $>26.12$ & 10 & 8 & \\
\hline \multirow{2}{*}{5.} & F0 (Hz) & $<210$ & 10 & 8 & \multirow[b]{2}{*}{1} \\
\hline & & $>210$ & 9 & 8 & \\
\hline
\end{tabular}

* Fisher's exact test applied. 


\section{Discussion}

Endotracheal intubation was not advocated for general anaesthesia until 1910 by Elsberg. ${ }^{12}$ It has since become one of the most common procedures performed in modern medical practice. Since it is an invasive procedure the risk of voice is always there. Tubes may injure the tracheal lumen through direct toxic effect of their component material or by physical effect ${ }^{13}$. However Polyvinylchloride (PVC) which is known to leech toxic additives remains the most commonly used material for all purposes whether that is for short or long term intubation. The cuffed tube of the same material was used in all the case of our series. Pressure at the interface between the wall of the endotracheal tube and the soft tissue of the larynx is the fundamental cause of mucosal ischemia. Capillary perfusion pressure is the critical factor in mucosal ischemia injury. In areas where mucosa is closely adherent to the cartilage, much lower intra cuff pressure can occlude the blood flow ${ }^{14}$. When ever capillary perfusion ceases, an ischemic injury is initiated. This injury is superficial when the time course is short ${ }^{15}$. Many other factors may play a role in modifying the intubation injury, but each is difficult to analyze as an independent variables. The most frequently mentioned includes: route of intubation (nasal vs. oral), shearing force (to-fro motion of the tube), presence of the nasogastric tube, pneumonia, steroid use, level of consciousness, hypotension and subsequent tracheostomy ${ }^{16,17}$.

Although many of the English literatures has mentioned the long term effect of endotracheal intubation in the trachea, very little attention has been paid to the vocal dysfunction following short term intubation ${ }^{8,18,19,20,21}$.

Voice is a co-ordinate output of the respiratory system, respiratory tract as well as function of the higher central nervous system. Aeromechanical energy produced by the lung displaces the different vocal tract structures, generating pressure behind the valves and produces the acoustic speech. Pulmonary function is an important factor to offer a vocal characteristics, such as intensity, pitch, stress syllable and phrases ${ }^{22}$ Restricted ventilation following general anaesthesia due to residual effects due to anaesthetic drugs, post operative pain following any abdomino-thoracic surgery or immediate post operative analgesia may alter the vocal characteristic ${ }^{23,24}$. To avoid all these effects on the acoustic characteristics we measured it after 48 hours of incubational anaesthesia.

Jitter, which is the cycle to cycle variation of fundamental frequency did not show any significant differences between the two groups $(\mathrm{P}=0.633)$. Changes in jitter can be a result of alteration of the intrinsic vibratory characteristics of the vocal fold mucosa or associated vocal tract system that participates in voice production.
These effects can result from deficits in vocal fold mobility, changes in glottic tension and alteration of the mucosa of the vibratory margin of the vocal fold ${ }^{2}$. But Hamadan and Beckford had observed significantly higher values for jitter. But their post intubational data measurement was done much earlier than ours, and this could be the reason for increased jitter values in their studes $^{18,20}$

Shimmer perturbation also correlated well with the jitter. The values of jitter did not change between the two groups. This is expected as values of both these variables increases as the signals become noisier or more irregular. Increase in perturbation is indicative of harsh or breathy voice. The value of $\mathrm{F}_{0}$ was reduced in our study, though it was not statistically insignificant. Slight increase in the jitter value may be considered here while interpreting the decreased value of $\mathrm{F}_{0}$. If the intubation had caused some oedema on the vocal folds, the vibrating folds may have had additional mass to render lesser $\mathrm{F}_{0}$. The other factor contributing to the decreased mean $\mathrm{F}_{0}$ is reduced phonatory effect by the subject consciously or unconsciously trying to protect their tender vocal folds, thereby reducing the vocal loudness and $\mathrm{F}_{0}{ }^{8}$. But this is not applicable in our study, as the acoustic characteristics were measured 48 hours of intubation ${ }^{25}$. On the other hand, Gleeson and many others have shown a consistent increase in $\mathrm{F}_{0}$ after intubation. Yonick et al conducted a study on 15 subjects undergoing cardiac surgery and found no significant changes in the mean $\mathrm{F}_{0}$. Only perturbation had increased ${ }^{26}$. Such differences in between similar type of studies need to be analyzed more cautiously as it may be due to methological differences.

HNR ratio is an average ratio of energy of the harmonic components in the range ratio $70-4500 \mathrm{~Hz}$ to the intraharmonic components energy in the range 1500$4500 \mathrm{~Hz}$. The amount of energy conveyed in the F0 and its harmonics, divided by the energy in the noise frequencies is the HNR. Since this parameter correlates with the perception of vocal roughness and thereby correlates with the $\mathrm{F}_{0}{ }^{28}$ we obviously did not observe any changes between the two groups.

In the study we compared analyzed the pre intubation data with the duration of intubation. Independent variables like cuff pressure and volume of the endotracheal tubes are not addressed here, but all the tubes were made up of PVC.

Acoustically, the post intubation voice was characterized by minimal decrease in the $\mathrm{F}_{0}$ Without consistent changes in perturbation and HNR. The result of this study indicates that short term intubation anaesthesia 
does not alter the acoustic characteristics. But general anaesthesia has multisystem effects, some of which can disturb the fine harmony necessary for normal voice production. These factors are potentially responsible for some changes that might occur in the phonation commonly seen immediately after the anaesthesia with endotracheal procedure.

\section{Conclusion}

During our evaluation it became evident that short term endotracheal anaesthesia did not alter the acoustic characteristics. Since vocal output is the multi factorial, other parameters that may affect the acoustic quality needs further consideration. However, additional and larger studies are required to reveal the potential of this technique.

\section{Acknowledgement}

We gratefully acknowledge the statistical assistance of Mr. Umesh Aryal, lecturer and statistician from the department of community medicine.

\section{References}

1. Hedden M, Ersoz CJ, Donelly WH et al. Laryngotracheal damage after prolong use of endotracheal tubes in adults. JAMA. 1969;207: 703-8.

2. Hilding AC. Regeneration of respiratory epithelium after minimal surface trauma.Ann. Otol. Rhinol. Laryngol. 1965;74:903-14.

3. Lindhom CE. Prolonged endotracheal intubation. Acta Anesthesiol Scand. 1970;33:115.

4. Priebe H, H. Henke W, Hedley-Whyte J. Effects of tracheal intubation laryngeal acoustic waveforms. Anes Anal. 1988;67:219-27.

5. Bishop MJ, weymuller EA, Fink BR.Laryngeal effects of prolong intubation. Anesth. Anlg. 1984;63:335-42.

6. Cooper JD, Grillo HC. Experimental production and prevention of injury due to cuffed tracheal tubes.Surg. Gynecol. Obstet.1969;129:1-235.

7. Horii Y, Fuller BS Selected acoustic characteristics of voices before intubation and after extubation. J Speech Lang Hear Res. 1990;33: 505-510.

8. Horii Y, Fuller BS. Accoustic characteristics of voices before and after intubation. Asha.1987:29:91.

9. Yonick TA, Minifie FDReich AR et al. Glottal source alteration associated with indotracheal intubation. Asha.1988;30: 96.

10. De Bodt MS, Wuyts FL, Heyning PH et al. Test-retest study of the GRBAS scale: influence of experience and profession background on perceptual rating of voice quality.J of Voice.1997;11(1):74-80.

11. De Fellipe, Grillo MHMM, Grechi TH. Standardization of of acoustic measure of normal voice patterns. Rev Bras Otorhinolaryngol.2006;72(5): 654-9.

12. Beckford NS, Mayo R, Wilkinson A III, Tierney M. Effects of short-term endotracheal intubation on vocal function. Laryngoscope. 1990; 100: 331-6.

13. Weymuller EA Jr. Laryngeal injury from prolong intubation. Laryngoscope.1988;98:1-15.

14. Nordin U Lindholm, CE, Wolgast, M. Blood flow in rabbit tracheal mucosa and the effect of tracheal intubation. Acta Anesthesiol. Scand.1977;21:81-94.

15. Hilding AC. Laryngotracheal damage during intra-taracheal anesthesia. Demonstration by staing the unfixed specimen with methylene blue, Ann Otol. 1971;80: 565-80.

16. Kirchner JA, Sasaki CT. Fusion of the vocal cords following intubation and tracheostomy. Trans. Am. Acad. Ophthmol. Otolaryngol. 1973;77:88-91.

17. Bogdasarian, RS, Olson NR. Posterior glottis laryngeal stenosis. Otolaryngol. Head neck surg. 1980;89:765-72.

18. Hamadan AL, Sibai A, Rameh C et al. Short term effects of endotracheal intubation on voice. $\mathrm{J}$ of voice. 2007;21(6):762-8.

19. Peppard S, Dickens J. Laryngeal injury following short term intubation. Ann Otol Rhinol Laryngol. 1983;92:327-30.

20. Beckford N, Mayo R, Wilkinsom A et al., Effects of short term endotracheal intubation on vocal function. Laryngoscope. 1990;100:3316.

21. Blanc V, Trembley G, Normand A. The complication of tracheal intubation: a new classification eith the review of the literature. Anes Anal. 1974;53:202-13.

22. Hixon TJ. Respiratory function in speech and song. Boston:Little Brown Boston; 1987.

23. Tolics $\mathrm{MD}$ et al. Lung collapse and gas exchange during general anesthesia: Effect of spontaneous breathing, muscle paralysi, and positive end expiratory pressure. Anesthesiology.1987;66:157.

24. Cartwright MB et al., Ventillation depression related to Plasma phentanyl concentration during and after anesthesia in humans. Anesth Analg. 1983;62:966-974. 
25. Gleeson MJ, Fourcin Aj. Clinical analysis of laryngeal trauma secondary to intubation. J R Soc Med. 1983;76:928.

26. Yonick T, Reich A, Minifie F et al. Acoustic effects of endotraccheal intunation. J Speech Hear Disorderd. 1990;55:427-33.
27. Smits I, Ceuppens P, de Bot MS. A comparative study of acoustic measurements by means of Dr. Speech computerizeds speech lab. 2005;19(2): 187-96.

28. Woodson GE, Cannito M, Voice analysis. In Cummings eds. Otolaryngology-head and neck surgery. 3rd ed. USA: St. Louis-Mosby-year book; 1988.p.1876-90. 